\title{
Transnacionalismo. Emergencia y fundamentos de una nueva perspectiva migratoria
}

\author{
Cristina Blanco F. de Valderrama \\ Universidad del País Vasco/Euskal Herriko Unibersitatea \\ Departamento de Sociología II \\ cristina.blanco@ehu.es
}

\section{Resumen}

El desarrollo de nuevas tecnologías en el ámbito de los transportes y de las comunicaciones tiene grandes repercusiones en la gestación, la duración y las modalidades de los movimientos migratorios internacionales. El abaratamiento de los precios de los transportes, su mayor rapidez, la incorporación de nuevas tecnologías en la comunicación, la telemática, etc. posibilitan que, hoy día, cualquier parte del mundo esté «cerca» del resto. Las distancias físicas se acortan, los movimientos se multiplican y el contacto virtual con los otros se hace posible en cualquier rincón del planeta.

Esta situación tendrá consecuencias no sólo sobre la cantidad de movilidad de gentes a través de las actuales fronteras, sino también sobre las características mismas de las migraciones y sobre los propios conceptos de «origen» y de «destino», así como sobre sus relaciones a través de las poblaciones migrantes.

Ante las nuevas circunstancias de movilidad, se hace necesario revisar los tradicionales paradigmas de integración de los inmigrantes en las sociedades receptoras. En este contexto, destaca la emergencia de una nueva perspectiva o enfoque migratorio: el transnacionalismo.

El presente artículo tiene como objetivo presentar las líneas maestras de esta nueva perspectiva de la mano de los principales sociólogos y antropólogos que defienden su validez e idoneidad para abordar nuevas formas de movilidad internacional.

Palabras clave: migraciones internacionales, transnacionalismo, comunidades transnacionales, actividades transnacionales, integración.

\section{Abstract. Transnationalism. The emergence of a new migratory approach}

The growth of new technologies in the area of the transport and of the communications has big repercussions in the gestation, duration and modalities of the migratory international movements. The cheapening of the prices of the transport, their greater rapidity, the incorporation of new technologies in the communication, the telematic, etc. They make possible that today any part of the world is «nearby» of the rest. The physical distances are slow, the movements multiply and the virtual contact with the others it makes possible in any part of the world.

This situation will have consequences not only on the quantity of mobility of people across the current borders, but on the characteristics themselves of the migrations and on the concepts of "origin» and of «destiny», as well as on their relations among the migrant populations. 
In the new circumstances of mobility, it becomes necessary to check the traditional paradigms of integration of the immigrants in the host societies. In this context stands out the emergency of a new perspective or migratory approach: the transnationalism.

This article present the main lines of this new perspective of the hand of the principal sociologists and anthropologists who defend its validity and suitability to approach new forms of international mobility.

Key words: international migrations, transnationalism, transnational communities, transnational activities, integration.

\section{Sumario}

Introducción Transnacionalismo como campo de estudio

La emergencia de una nueva perspectiva migratoria: el transnacionalismo Líneas futuras de investigación

Los conceptos y sus debates En conclusión

Dimensiones y tipologias Bibliografía

Sobre la novedad del fenómeno transnacional

\section{Introducción}

La creciente movilidad internacional es un hecho constatado en un mundo globalizado como el que nos ha tocado vivir. No sólo las personas se mueven, también lo hacen los capitales, las empresas, las maneras de hacer, las ideas... Todo este movimiento creciente tiene su gran aliado en los importantes avances tecnológicos de los últimos tiempos, por medio de los cuales se consigue un abaratamiento importante de los transportes, su mayor rapidez, la incorporación de nuevas tecnologías en la comunicación, la telemática, etc. Todo ello hace que, hoy día, cualquier parte del mundo tenga posibilidad de estar "cerca» del resto. Las distancias físicas se acortan, los movimientos se multiplican y el contacto virtual con los otros se hace posible en cualquier rincón del planeta.

El acortamiento de las distancias físicas posibilita que éstas puedan ser transitadas con mayor frecuencia que en épocas anteriores; por otro lado, las nuevas tecnologías nos habilitan para establecer relaciones virtuales con cualquier parte del mundo, lo que permite el mantenimiento de lazos y de relaciones continuas, estables y frecuentes con nuestro país, nuestra familia, nuestros amigos y nuestros compatriotas, aunque hayamos emigrado muy lejos de ellos.

Esta situación, sin duda, tendrá consecuencias no sólo sobre la cantidad de movilidad de gentes a través de las fronteras actuales, sino también sobre las características mismas de las migraciones y sobre los propios conceptos de «origen" y de "destino», así como sobre sus relaciones a través de las poblaciones migrantes. 
Por otra parte, se advierte una tendencia a la concentración residencial de los inmigrantes en las sociedades receptoras. El proceso de segregación residencial no es nuevo, pero sí lo son las posibilidades de establecer verdaderas comunidades de origen en los espacios urbanos de las sociedades de destino, alentadas por el incremento de relaciones con la sociedad de origen. Esta cada vez más tupida red de relaciones se alimenta de los viajes frecuentes entre origen y destino, de las actividades transnacionales que enlazan ambos lados de la red migratoria y de los contactos virtuales de los migrantes con sus familias, amigos y compatriotas que se quedaron en origen.

Somos conscientes de que, ante las nuevas circunstancias de movilidad humana en nuestro mundo contemporáneo, se hace necesario revisar los tradicionales paradigmas de integración de los inmigrantes en las sociedades receptoras.

La revisión de los viejos paradigmas de integración debe ir precedida, sin embargo, por un mejor conocimiento de los fenómenos que los hacen obsoletos, por lo menos en algunos contextos y situaciones, y que se refuerzan entre sí: el transnacionalismo como nueva forma de movilidad, por un lado, y la configuración de enclaves étnicos ${ }^{1}$ como modelo de asentamiento residencial de los inmigrantes, por otro.

En este artículo pretendemos abordar el primero de ellos, ofreciendo una aproximación a la nueva perspectiva transnacional de la mano de algunos de los principales sociólogos y antropólogos que se han hecho eco de lo que consideran un nuevo fenómeno social de consecuencias desconocidas, pero que, por su novedad, se encuentra en una situación de notable ambigüedad e indefinición.

\section{La emergencia de una nueva perspectiva migratoria: el transnacionalismo}

Los movimientos y las actividades transnacionales, como las mismas migraciones en general, no constituyen hechos novedosos en la historia de la humanidad y, sin embargo, el transnacionalismo (¿o quizá deberíamos decir la transnacionalidad?) está emergiendo en los últimos tiempos como un auténtico nuevo fenómeno social y, por ende, como justificada nueva materia de estudio (Portes, Guarnizo y Landlot; 1999: 219).

La aparente paradoja no debe engañarnos; en realidad, no se trata de ocultar bajo nuevos términos viejas realidades explicadas, reinventando, con ello, todo

1. No debe confundirse el enclave étnico con el gueto, a pesar de que ambas figuras constituyen formas concentradas de residencia de inmigrantes en las sociedades receptoras. Sin embargo, el término gueto se reserva para aquellas concentraciones residenciales que son fruto de procesos de exclusión social generados desde la sociedad receptora hacia las poblaciones inmigrantes, resultando, con ello, procesos de segregación espacial. Por su parte, el enclave étnico es una concentración residencial derivada del deseo de los propios inmigrantes de mantenerse unidos con el fin de preservar su cultura, su identidad y sus formas de desarrollo económico y social, manteniendo, además, vínculos estrechos con las sociedades de origen (economía étnica en destino, remesas, actividades de codesarrollo, participación política en origen, etc.). 
lo ya dicho. La multiplicidad de nuevas formas de relaciones sociales intrínsecamente unidas al avance de las nuevas tecnologías, de los sistemas de transportes y de las comunicaciones, es un hecho empíricamente contrastado. Como dirán Portes, Escobar y Walton en un reciente trabajo (2006), el peso de la evidencia empírica constituye una sólida prueba del carácter novedoso de estas prácticas (transnacionales) y de su importancia estructural para las regiones emisoras y para las propias comunidades migrantes.

La nueva perspectiva del transnacionalismo — dirá Caglar - ofrece una nueva óptica que permite hacer visible la creciente intensidad y extensión de los flujos circulares de personas, bienes, información y símbolos alcanzados por las migraciones internacionales. Permite analizar cómo los migrantes construyen y reconstruyen sus vidas de forma simultánea en más de una sociedad (Caglar, 2001: 607).

Cierto es que, durante la última década del siglo XX, se ha utilizado con profusión el término transnacionalismo y sus derivaciones transnacionales. Esta utilización ha sido particularmente profusa en el ámbito de las migraciones internacionales, hasta el punto de acuñarse un nuevo término, transmigración, no compartido por todos. Este uso, y a veces abuso, del término transnacional no siempre ha sido debidamente fundamentado.

Ante la notable ambigüedad, indefinición y/o uso indiscriminado del término, algunos autores han rechazado de plano la utilización de este concepto como descriptor de un fenómeno nuevo ${ }^{2}$.

En este sentido, Vertovec (2004: 3-4) hace un recuento de las críticas que usualmente se hacen al enfoque transnacional:

- Confusión conceptual y uso abusivo: utilización del término transnacionalismo como sinónimo de internacional, multinacional, global o diaspórico. Se sugiere también con mucha frecuencia que todos los migrantes están involucrados en procesos transnacionales.

- Antigüedad / novedad: de esta perspectiva se deriva la idea de que todas las actividades transnacionales de los inmigrantes son nuevas.

- Búsqueda de la variable dependiente: los investigadores sólo tratan los casos en los que se encuentran actividades transnacionales, pero no se suele hacer mención de los casos en los que tales actividades no son encontradas.

2. Es el caso de David Fitzgerald, quien considera que tal término debería ser desterrado de las investigaciones sobre migraciones internacionales por su inutilidad para describir los diferentes procesos de adscripción identitaria de los migrantes transnacionales. Él encuentra más útil la diferenciación entre nacionalismo dual y nacionalismo a distancia. Esta diferenciación permite superar la negación de las posibilidades de asimilación entre los migrantes transnacionales que augura la perspectiva transnacional tal y como se presenta en la actualidad (Fitzgerald, 2000, 2004). En un artículo reciente escrito con Waldinger, Transnationalism in Question (2004), insiste en la idea de que, si existe el transnacionalismo, éste dista mucho de consistir en procesos liberadores y autónomos de la sociedad civil que construyen espacios sociales independientes de la economía mundial y de la política nacional. 
— ¿Trans-qué?: no se hacen las debidas distinciones entre los procesos y los fenómenos trans-nacionales, trans-estatales y trans-locales. En realidad, no todo es lo mismo.

- Transnacionalismo versus asimilación (o versus multiculturalismo): se establecen falsas dicotomías entre estos términos, en vez de analizar las verdaderas relaciones que puede haber entre ellos.

- Determinismo tecnológico: ¿son las formas contemporáneas de migración transnacional mera consecuencia de las comunicaciones en tiempo real y del abaratamiento de los transportes?

- No todos los migrantes son transnacionales: hay una gran variedad de prácticas que atraviesan fronteras y no por ello deben ser consideradas transnacionales.

- Limitación generacional: ¿̨as actuales prácticas transnacionales, serán continuadas por las siguientes generaciones de inmigrantes?

El propio autor reconoce que estas críticas tienen su fundamento, pero que, más que negar la existencia del transnacionalismo, evidencian la necesidad de acotar su uso y definir su contenido.

Así pues, por un lado nos encontramos, en la década de 1990, con una utilización arbitraria y excesiva del término transnacional, que identifica como tal todo aquello que hasta hacía poco era simplemente «inter-nacional». Por otro lado, un grupo de científicos sociales se muestran altamente escépticos en relación con la existencia del transnacionalismo como un fenómeno verdaderamente novedoso.

Frente a estas posturas, un nutrido grupo de investigadores, sociólogos y antropólogos, fundamentalmente, son conscientes de que, bajo el amparo de la globalización y sus derivados, se están gestando nuevas realidades sociales que atienden a un denominador común que podría denominarse "transnacionalismo»; realidades que difieren de los ámbitos internacional y/o multinacional ${ }^{3}$ y que, sin duda, tendrán, en un futuro no lejano, implicaciones económicas, políticas y sociales verdaderamente importantes ${ }^{4}$. Conscientes también de lo etéreo de este "emergente campo social», se apresuran a identificar la necesidad de trabajar seriamente en torno a él, convirtiéndolo en una verdadera área de investigación social. Para ello, son varios los pasos que hay que dar: identificar el fenómeno, delimitarlo, definirlo, describirlo estableciendo

3. Portes, Escobar y Walton (2006) consideran que las actividades transnacionales que realizan los migrantes y la sociedad civil «desde abajo» (Guarnizo y Smith, 1998) deben distinguirse de las internacionales (realizadas por gobiernos e instituciones de carácter nacional que tienen como base para la acción al Estado nación) y de las multinacionales (realizadas por organizaciones, iglesias, empresas, corporaciones que operan en diferentes países y cuya base para la acción es la actividad que ejercen).

4. En este sentido, el texto de Vertovec de 2004, Trends and Impacts of Migrant Transnationalism, lo dedica a avanzar las posibles implicaciones de este nuevo fenómeno sobre las sociedades de emisión y recepción de este tipo de migración, tanto en lo que se refiere a las relaciones sociales, como a las políticas y a las económicas. 
componentes y dimensiones, operacionalizarlo y proceder a su investigación empírica en cada una de sus manifestaciones (Portes y otros, 1999).

\section{Los conceptos y sus debates}

La situación del transnacionalismo como campo de investigación social aún joven se manifiesta en los intensos debates que atraviesan la literatura en torno al fenómeno en los últimos quince años. Estas discusiones en el seno de la comunidad científica, que incluyen la propia existencia del transnacionalismo como nuevo fenómeno social, son especialmente intensos cuando se avanzan sus posibles consecuencias sobre las sociedades actuales y el orden social contemporáneo. Así, unos consideran que las actividades transnacionales se ubican en un "tercer espacio» desterritorializado, ajenas a localidades y naciones preexistentes y, por lo tanto, con capacidad para erosionar y derrumbar al actual sistema del Estado nación, al cual trascienden (Kearney, 1991, 1995; Kastoryano, 2002). Algunos incluso van más allá, entendiendo que los migrantes transnacionales realizan una labor «liberadora» precisamente por eludir las limitaciones actuales del statu quo. A nivel más local, esta supuesta ausencia de referentes sociales específicos de los transmigrantes, según unos, o el mantenimiento y la perpetuación de la identidad de origen, según otros, son motivos más que suficientes para temer por la falta de integración de los inmigrantes en las sociedades receptoras. Esta sensación es especialmente intensa en el contexto norteamericano, donde el proceso de asimilación es entendido por muchos como el único verdaderamente eficaz para eliminar la discriminación racial y la exclusión social. Los inmigrantes inmersos en redes y actividades transnacionales, en continuo contacto referencial y práctico con sus comunidades de origen, tendrían verdaderas dificultades para asimilarse en las sociedades receptoras.

Otros, sin embargo, mantienen posturas contrarias, argumentando contra cada uno de los aspectos anteriormente mencionados: las actividades transnacionales no se realizan ajenas a las limitaciones y a las oportunidades de las localidades y/o naciones de origen y destino, ni pueden evitar las normativas que organizan las vidas en uno y otro referente local concreto; los migrantes transnacionales, en muchos casos, lejos de contravenir los imperativos económicos, legales, de dominación... existentes, lo que hacen es perpetuarlos bajo nuevas formas y condiciones 5 (Smith y Guarnizo, 1998). Por su parte, y en referencia al asedio al Estado nación, algunos autores indican que el transnacionalismo no acabará con esta manera de organización político-administrativa, si bien obligará a redefinir las relaciones entre Estado y Nación (Kastoryano, 2003). Y en lo que se refiere al reto que el transnacionalismo plantea a la integración y asimilación, algunos autores defienden que el transnacionalismo no es incom-

5. Es el clásico ejemplo de las comunidades chinas y su manera de ejercer actividades económicas en los países de asentamiento, más propias de una economía capitalista del siglo XIX que de los actuales estados sociales y de derecho de los países desarrollados en que se establecen. 
patible con la asimilación y que, en cualquier caso, aún generando nuevas comunidades panétnicas (Werbner, 2002) o comunidades imaginadas (Anderson, 1983), el punto crucial es saber si estos procesos se mantienen en las segundas generaciones y en las posteriores, lo cual parece bastante improbable (Lucassen, 2004).

Éstas son sólo las líneas directrices de los debates generados en los últimos años por el transnacionalismo, un fenómeno de nuevo cuño sobre el que aún queda mucho por investigar. No obstante, es necesario empezar a ubicarlo en sus principales coordenadas conceptuales y metodológicas, tal y como se encuentra en este momento.

La acepción inicial más conocida del término transnacional se debe a las antropólogas Glick-Schiller, Basch y Szanton-Blanc, quienes, en un trabajo publicado en 1992, indicaban que la emergencia de espacios sociales transnacionales que unen de forma especial las sociedades de origen y de destino de los migrantes representan una ruptura con el pasado. La desconexión con el origen ya no es necesaria; antes bien, en el mundo contemporáneo, los «transmigrantes» mantienen, construyen y refuerzan múltiples lazos que les unen a sus lugares de origen, generando, de este modo, verdaderas «comunidades desterritorializadas». El transnacionalismo sería el conjunto de actividades creadas por los migrantes transnacionales que les permiten vivir de forma simultánea en dos comunidades diferenciadas. Transmigrantes serían, entonces, los inmigrantes que cotidianamente viven dependiendo de las múltiples y constantes interconexiones a través de las fronteras nacionales y cuyas identidades se configuran en relación con más de una nación Estado (Glick-Schiller, Basch y Szanton-Blanc, 1995).

Así concebido, y en la medida en que el concepto se oponía a los tradicionales modelos de asimilación americanos, el transnacionalismo obtuvo intensas críticas entre muchos científicos sociales. Ante estas reacciones, algunos investigadores que asumieron el transnacionalismo como una nueva realidad social emergente, decidieron limitar más y mejor el concepto. Así, Portes, Guarnizo y Landlot indicaban que era preferible limitar el concepto a ocupaciones y a actividades que requieren contactos sociales regulares y sostenidos en el tiempo a través de las fronteras nacionales (1999: 219), considerando, entonces, que los migrantes transnacionales son aquellas personas bilingües que se mueven con facilidad entre diferentes culturas, frecuentemente mantienen hogares en dos países y persiguen intereses económicos, políticos y culturales que requieren su presencia en ambos (Portes, 1996).

Si bien los movimientos migratorios constituyen la base fundamental del transnacionalismo así entendido, las migraciones no son sinónimo de transnacionalismo. Éste último es un fenómeno que acompaña a una manera particular de migrar que no necesariamente es universal, ni siquiera en la actualidad. No todos los migrantes son «transmigrantes»; sólo lo son aquéllos que mantienen relaciones estrechas entre el origen y el destino, con lo que presentan una acusada dualización o bipolarización de sus vidas. Por ello, el hecho de que se envíen remesas a las familias en origen, o de que se realicen viajes al 
lugar de origen, no constituye, en sí mismo, un rasgo de transnacionalismo. Se requiere regularidad y mantenimiento en el tiempo de las actividades transnacionales para que éstas tengan efectos reales sobre los sujetos migrantes y sus sociedades de origen y destino, con lo que se generan, así, verdaderas comunidades transnacionales. De este modo, las actividades que realizan los inmigrantes entre sus localidades de origen y destino (económicas, de envío de remesas, políticas, culturales, comunicativas, financieras...) adquieren un carácter transnacional en sentido estricto cuando éstas son regulares y sistemáticas, generando un verdadero canal interactivo entre ambos lugares: el espacio transnacional.

El espacio transnacional, según algunos, no constituye un espacio físico territorializado y concreto. Es precisamente la desterritorialización lo que caracteriza a las actuales comunidades transnacionales y las diferencia de otros grupos de migrantes más antiguos que también presentan características transfronterizas, como es el caso de las diásporas ${ }^{6}$. Otros, como ya hemos indicado anteriormente, consideran que la desterritorialización de las actividades transnacionales constituye un mito irreal y que, por tanto, los espacios transnacionales se ubican en territorios concretos formados por las localidades o los países de origen y los de destino que unen las redes transnacionales. Si bien las prácticas transnacionales se extienden más allá de dos o tres territorios nacionales — comentan Smith y Guarnizo- ellas se construyen dentro de los límites de relaciones sociales, económicas y políticas específicas, los cuales mantienen a todos unidos por los intereses y los significados que se entienden compartidos (Smith y Guarnizo, 1998: 13).

No obstante, es posible que ambas versiones sobre la territorialización de los espacios transnacionales puedan ser compatibles, según el concepto de «espacio» que estemos manejando (territorial o social). Si consideramos al espacio transnacional como el soporte cuasi físico por el que transitan los bienes, los capitales, los votos, las comunicaciones, las personas..., éste sí puede considerarse perteneciente a ámbitos locales concretos y, efectivamente, estar sometido a normas, limitaciones y posibilidades que las sociedades de origen y destino han establecido para sí. Se trata de una conceptualización del espacio como soporte práctico de relaciones y actividades que se realizan entre límites territoriales precisos.

Pero hay otras dimensiones del «espacio» que la percepción más práctica suele no tener en cuenta. Una de ellas es la dimensión simbólica que adquieren las relaciones prácticas entre gentes y lugares, estableciendo lazos de identidad, afecto, reconocimiento y solidaridad entre ellos. Este enfoque, más social, queda recogido en la definición que realiza Faist en 1999 sobre espacios sociales transnacionales: espacios sociales transnacionales son combinaciones de

6. Las diásporas constituyen uno de los pocos ejemplos de comunidades transnacionales de gran antigüedad. A diferencia de las comunidades actuales, las diásporas siempre tenían como referencia un territorio, pasado, presente o futuro, en el que la comunidad dispersa por el mundo establecía su origen o su razón de ser. 
lazos sociales y simbólicos, posiciones en redes y organizaciones, y redes de organizaciones que pueden encontrarse presentes en al menos dos lugares geográfica e internacionalmente distintos (Faist, 1999b: 40).

En su conjunto, hablamos de espacios territoriales a través de los cuales transitan bienes, símbolos, votos, capital, relaciones sociales, etc., formando verdaderas redes que vinculan a los migrantes con sus comunidades de origen y sus lugares de asentamiento, posibilitando, con ello, la generación de espacios sociales transnacionales a los cuales se adscriben comunidades transnacionales.

El concepto de comunidad transnacional tampoco está demasiado claro, pues algunos autores entienden por tal el conjunto de personas que quedan unidas por lazos transnacionales y que pertenecen al grupo de migrantes, a las comunidades de origen y a las comunidades de destino. Faist escribe que las comunidades transnacionales que se forman a partir de los movimientos transnacionales se caracterizan por ser una situación en la cual los migrantes, las personas que se quedan en el país de origen y los nacionales de los países anfitriones, están conectados por lazos sociales y simbólicos densos y fuertes, los cuales, a través del tiempo y del espacio, configuran redes y circuitos en los países de origen y destino, teniendo como base la solidaridad (Faist, 1999a: 10).

Otros, sin embargo, se refieren sólo a los grupos de inmigrantes procedentes de un mismo país que se asientan en una misma comunidad de destino, hibridando elementos culturales de las dos comunidades gracias a la circulación constante de bienes materiales, bienes culturales y personas entre ambos lugares, pero permaneciendo unidos como grupo diferenciado del resto de la población del lugar de residencia ${ }^{7}$.

Por último, se podría establecer un concepto de comunidad transnacional que superara incluso la dualización territorial de origen y destino, incorporando a la comunidad transnacional a aquellos inmigrantes del mismo origen que residan en diferentes países, pero que se encuentren interconectados por relaciones virtuales gracias a la telemática y a los medios de comunicación (chats, foros, páginas web de carácter nacional, emisoras de radio y televisión de carácter nacional que emiten en diferentes países y generan espacios de comunicación en directo...). Entendemos que este último aspecto, el de la conectividad, ha sido poco desarrollado, por lo que escasamente se ha reparado en sus potencialidades para conformar grupo y mantener identidades.

\section{Dimensiones y tipologías}

Bajo el calificativo de transnacional puede acomodarse una diversidad de actividades. En su artículo introductorio al volumen especial sobre transnaciona-

7. Este tipo de consideraciones es muy frecuente entre los antropólogos que realizan estudios de caso. Véase el ejemplo de Zevallos sobre Desplazamiento y transnacionalismo en la construcción de una identidad cultural andina en los EEUU (2004). 
lismo de la revista Ethnic and Racial Studies (vol. 22, no 2), Portes y colaboradores realizan una primera tipologización de actividades transnacionales basándose en dos dimensiones: el sector en el que se enmarcan y su nivel de institucionalización. Se llega, así, al cuadro que reproducimos a continuación (figura 1) y que contiene unos cuantos ejemplos de actividades transnacionales en base a estas dos dimensiones tenidas en cuenta.

Según los autores, existen tres grandes tipos de transnacionalismo según el sector de la actividad transnacional: el transnacionalismo político, el económico y el sociocultural.

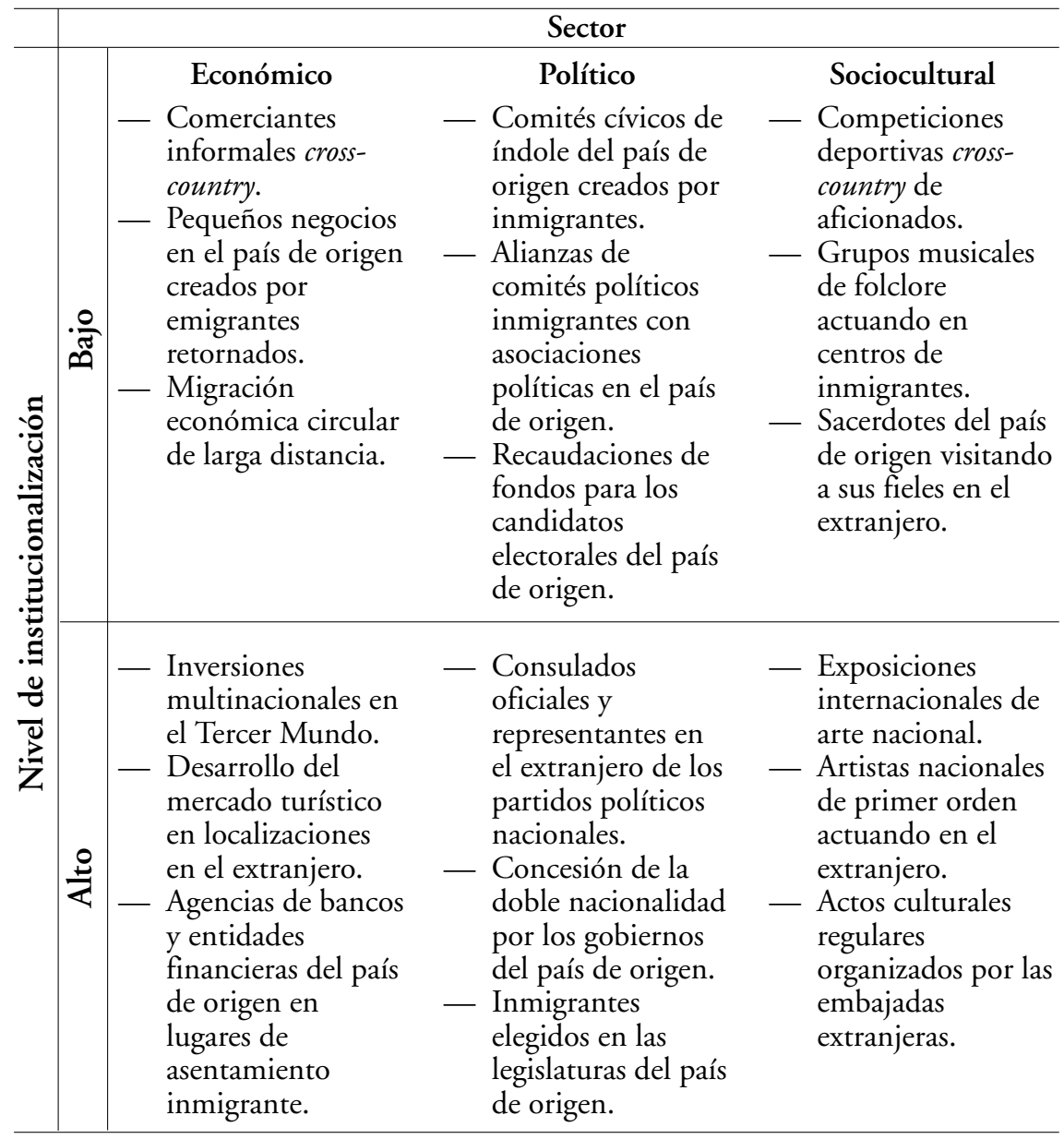

Figura 1. Tipos de actividades transnacionales. Fuente: Portes, Guarnizo y Landlot, 1999: 222. 
Así, por un lado, contamos con las actividades económicas de los empresarios transnacionales, que movilizan sus contactos a través de las fronteras en busca de proveedores, capital y mercados. Por otro lado, se encuentran las actividades políticas de partidos oficiales, funcionarios gubernamentales o líderes comunitarios cuyos objetivos son conseguir poder político e influencia en los países de origen, en los de destino o en ambos. Por último, existe una tercera categoría de actividades más amplia que comprende a múltiples iniciativas socioculturales cuyo principal objetivo es reforzar la identidad nacional en el extranjero.

Una segunda dimensión, cuya consideración suele resultar muy útil para la operacionalización del fenómeno y su análisis empírico, es el tipo de actor que lleva a cabo la actividad transnacional: si se trata de un actor institucionalmente poderoso (estados y corporaciones multinacionales) o si se trata de iniciativas llevadas a cabo por los propios inmigrantes y sus compatriotas en origen. Esta dimensión ha sido tratada por Guarnizo como transnacionalismo "desde arriba» y "desde abajo» según el tipo de actor que lo lidere.

Las actividades transnacionales "desde arriba» son bastante bien conocidas en el mundo actual, mientras que las realizadas "desde abajo» son el objeto de estudio más importante por parte de los investigadores del transnacionalismo, precisamente por su desconocimiento y sus consecuencias futuras.

\section{Sobre la novedad del fenómeno transnacional}

Ciertamente a lo largo de la historia han existido actividades transnacionales importantes, pero, a juicio de Portes, a estas actividades les ha faltado la regularidad, la rutina y la masa crítica que caracterizan a los ejemplos contemporáneos de transnacionalismo. Aun cuando haya podido haber casos de verdadero transnacionalismo en épocas anteriores, éstos han sido excepcionales. El transnacionalismo contemporáneo — dirán los autores - corresponde a un período diferente en la evolución de la economía mundial y a un conjunto diferente de respuestas y de estrategias desarrolladas por la gente en condiciones de desventaja ante la lógica dominante. En ello reside la importancia de su emergencia (Portes, Guarnizo y Landlot, 1999: 227).

A pesar de la no novedad de las actividades transnacionales y de la existencia previa de ejemplos claros de comunidades transnacionales en épocas históricas previas, Portes y colaboradores mantienen la idea de que el transnacionalismo constituye un nuevo campo de investigación social. Lo que constituye la verdadera originalidad del fenómeno y, por tanto, justifica su consideración como nuevo tema de investigación, es la elevada intensidad de los intercambios, los nuevos modos de transacción y la multiplicación de actividades que requieren viajes y contactos a través de las fronteras nacionales de forma sostenida en el tiempo (Portes, Guarnizo y Landlot, 1999: 219).

Evidentemente, los avances tecnológicos y la globalización han posibilitado la multiplicación de los viajes circulares, la conectividad virtual entre las personas a través de las fronteras nacionales y la realización de actividades 
comerciales, políticas y culturales por encima de los límites fronterizos actuales a unos niveles antes nunca conocidos. El proceso de globalización, en el que se enmarca el transnacionalismo — dirá Doña — refleja la emergencia de redes interregionales y sistemas de interacción e intercambio. Así, el alcance y la densidad de su interconectividad global y transnacional construye complejas redes de relaciones entre comunidades, estados, instituciones internacionales, organizaciones no gubernamentales y corporaciones multinacionales que constituyen el orden mundial. Las interacciones mencionadas han generado una nueva tendencia en la migración internacional llamada «movimientos transnacionales» (Doña, 2004).

No parece descabellado pensar que quizá el incremento de esta masa crítica pueda tener implicaciones sociales importantes y diferentes de las que hasta ahora han tenido los escasos y excepcionales casos de transnacionalismo. La intensificación del fenómeno (que no universalización todavía) es lo realmente novedoso del transnacionalismo, y las potencialidades de gestación de nuevos procesos sociales lo que justifica, a nuestro juicio, la consideración del transnacionalismo como un nuevo campo de estudio en el área de las ciencias sociales.

\section{Transnacionalismo como campo de estudio}

Es ya nutrida la generación de autores e investigadores que han tomado el transnacionalismo migratorio como objeto de estudio y se han dedicado a definir su contenido, a operacionalizarlo y a investigar empíricamente su existencia, sus manifestaciones, tipologías y consecuencias ${ }^{8}$, generando un campo de estudio emergente y vigoroso. No obstante, queda aún mucho por hacer, sobre todo en el ámbito europeo. La proliferación de lazos transnacionales «desde abajo» a través del mundo — escriben Guarnizo, Portes y Haller- es un fenómeno de gran significación y, sin embargo, ha recibido escasa atención en la literatura sociológica (Guarnizo, Portes y Haller, 2003: 1212). Aún así, hay ya numerosos estudios empíricos sobre casos concretos de redes, actividades y/o comunidades transnacionales.

Los estudios empíricos sobre el transnacionalismo contemporáneo han girado sustancialmente en torno a las dimensiones políticas (Guarnizo, Portes y Haller, 2003; Landlot, 2003) y económicas (Guarnizo, 2003; Landlot, 2001; Taylor, 1999; Portes, Haller y Guarnizo, 2002) del fenómeno, mientras que el debate más ideológico se ha centrado sobre la potencial incidencia del trans-

8. Steven Vertovec, Alejandro Portes, Luis Guarnizo, Thomas Faist, Patricia Landlot, entre muchos otros. Un caso especial que ejemplifica la importancia de este nuevo campo de estudio es la creación de un Programa específico de investigación sobre comunidades transnacionales de cinco años de duración (1997-2003) en el seno del Instituto de Antropología Social y Cultural (ISCA) de la Universidad de Oxford. En este programa, dirigido por Vertovec, se desarrollaron diversos proyectos de investigación, eventos y publicaciones en torno a este tema y sus ramificaciones sociales, culturales, políticas, económicas e identitarias. 
nacionalismo en los procesos de integración de estos inmigrantes en las sociedades receptoras, esto es, la supuesta dicotomía entre transnacionalismo y asimilación (Guarnizo, Portes y Haller, 2003).

El contexto más usual de las investigaciones sobre transnacionalismo es, sin duda, Estados Unidos, donde se toman como comunidades transnacionales más frecuentes las procedentes de Latinoamérica, si bien también existen algunos estudios que toman como unidad de análisis las comunidades chinas (Mitchell, 1993; Ong, 1997). En este contexto, la estrategia investigadora más frecuente es la de determinar las características del fenómeno más que sus consecuencias o implicaciones. Se utilizan tanto metodologías cuantitativas, propias de la sociología, como cualitativas y etnográficas, más cercanas a la antropología.

En Europa, sin embargo, los estudios sobre transnacionalismo son mucho más limitados. Conscientes de ello, los responsables de la European Science Foundation organizaron en junio de 2003, en Portsmouth (Reino Unido), un workshop exploratorio en torno al transnacionalismo en la Unión Europea. En el documento publicado tras el evento se reconoce que, mientras en la década de 1990 los estudios sobre transnacionalismo han girado en torno a las conexiones sociales globales y transregionales, en la Unión Europea los estudios han permanecido enfocados exclusivamente en los ámbitos comunitarios de toma de decisiones y en las políticas domésticas nacionales. Europa está demasiado centrada en el Estado nación. La reunión sirvió, a juicio de sus organizadores, para mostrar la necesidad de avanzar en este nuevo campo de estudio que debe abarcar fenómenos que van más allá de la política comunitaria (ESF, Transnationalism in the European Union, Scientific Report, 2003).

La centralidad del Estado nación en Europa se materializa en la inquietud que genera la idea de que las actividades transnacionales, y la consiguiente formación de comunidades étnicas de origen extranjero, puedan erosionar el actual Estado nación. Así, los análisis europeos sobre transnacionalismo giran más en torno a esta posibilidad que a las propias actividades transnacionales. Los temas más recurrentes entre los estudiosos europeos son los de ciudadanía, nacionalismo e identidad étnica, y la preocupación principal es la incidencia del asentamiento de comunidades étnicas en tierras europeas sobre las identidades nacionales europeas y la configuración del Estado nación (la ciudadanía transnacional de Bauböck, 1994; Soysal, 1994, y Kastoryano, 2002, 2003).

\section{Líneas futuras de investigación}

Todo lo expresado anteriormente pretende ser un pequeño ejemplo de la indefinición en que actualmente se encuentra el transnacionalismo como objeto de estudio, lo que no deja de ser una situación propia de un fenómeno novedoso y emergente. A pesar de cierta ambigüedad y falta de definición y operacionalización de conceptos, lo dibujado en las páginas precedentes también da cuenta del gran dinamismo que presenta el estudio del transnacionalismo en todas sus vertientes y del intenso futuro que le espera como objeto de estudio 
por parte de los científicos y analistas sociales. Algunos autores ya avanzan las necesidades que deberían cubrirse en la investigación futura del transnacionalismo.

Smith y Guarnizo (1998), por ejemplo, enfatizan las cuestiones metodológicas desde las que abordar el fenómeno del transnacionalismo. Además de citar algunos aspectos temáticos que requieren una intensa investigación (como es el caso de la incidencia de las actividades transnacionales sobre las segundas y terceras generaciones), los autores inciden de manera especial sobre las necesidades epistemológicas y metodológicas del estudio del transnacionalismo. Dado el mayoritario enfoque antropológico de los estudios empíricos de caso, utilizando metodologías etnográficas y cualitativas en el estudio de comunidades concretas, Smith y Guarnizo estiman necesario reconducir la tendencia, con el fin de aprehender la complejidad del fenómeno y evitar algunos de los desatinos que ha producido la utilización única y abusiva de las metodologías etnográficas. El reto, entonces, es doble; esto es: integrar determinantes macro y micro en el análisis, y desarrollar una estrategia de investigación apropiada, capaz de capturar la complejidad del proceso transnacional (Smith y Guarnizo, 1998: 24).

Según estos autores, tres han sido, hasta ahora, las limitaciones y/o los errores producidos en el estudio del transnacionalismo que deberían ser superados en el futuro:

- La no utilización, y definición clara, de métodos de investigación adecuados; es necesaria la aproximación a varios niveles: macro, medio y micro.

- La confusión entre lo que son los propios hechos sociales con sus posibles consecuencias. En muchas ocasiones, se confunden procesos y conceptos, utilizando de forma intercambiable aquéllos que describen los procesos (por ejemplo: redes sociales transnacionales) y los que definen resultados o consecuencias de éstos (por ejemplo: comunidades transnacionales). Esto significa que se requiere un esfuerzo importante para definir conceptos y procesos que actualmente están mezclados y confusos, lo cual genera graves dificultades para la investigación empírica.

- No hay estudios comparativos sistemáticos que permitan extraer conclusiones de los estudios de caso. Los autores ponen varios ejemplos de estudios comparativos que serían necesarios: comparar prácticas del mismo grupo en diferentes contextos locales; comparar prácticas transnacionales en diferentes grupos que se encuentren en las mismas localidades, o comparar prácticas transnacionales de inmigrantes en diferentes regiones mundiales.

Por su parte, Vertovec (2004) insiste en la necesidad de abordar en el futuro las consecuencias a diferentes niveles que puede generar el transnacionalismo. Él mismo describe campos de análisis en tres dimensiones básicas a las que afecta el transnacionalismo: la sociocultural (la reorientación del habitus en el que se desenvuelven los individuos en las sociedades emisoras y receptoras), la política (reconfiguración de identidades, fronteras y órdenes establecidos) y 
la económica (influencia sobre el desarrollo). «Éstos y otros procesos dirá Vertovec - se producirán en el futuro próximo. Muchas pautas de transnacionalismo migrante y modos asociados de transformación como los citados se extenderán, se intensificarán y se acelerarán» (Vertovec, 2004: 55). Y aunque reconoce que las segundas y sucesivas generaciones pueden no mantener las orientaciones y las prácticas transnacionales de sus progenitores, indica que tales prácticas y orientaciones tendrán una influencia decisiva en sus identidades, intereses y actividades.

\section{En conclusión}

Tras esta breve exposición de la situación del transnacionalismo como área de investigación de las ciencias sociales aplicada a los movimientos migratorios internacionales, parece claro que existen fundamentos para considerarlo como un nuevo campo de investigación. Ya en 2003 Alejandro Portes elaboraba unas conclusiones al monográfico especial de International Migration Review dedicado a la nueva perspectiva transnacional, en las que establece cinco evidencias empíricas de la existencia del transnacionalismo como nuevo campo de estudio y en torno a las cuales existe un cierto grado de consenso (Portes, 2003: 875-892):

- El transnacionalismo es una perspectiva novedosa, no un fenómeno nuevo.

- El transnacionalismo es un fenómeno de las bases.

- No todas las migraciones internacionales son transnacionales.

- El transnacionalismo inmigrante tiene consecuencias macrosociales.

- El grado y las formas de transnacionalismo varía según los contextos de salida y recepción.

Entendemos que el transnacionalismo está especialmente relacionado con los procesos de integración de los inmigrantes en las sociedades receptoras en lo que se refiere a sus implicaciones, y también especialmente relacionado con las nuevas formas de asentamiento residencial de carácter étnico. Y es precisamente en estas relaciones estrechas con ambos procesos, de asentamiento residencial y de integración, fruto de la extensión sin precedentes de las actividades transnacionales, en donde, a nuestro juicio, reside la verdadera potencialidad de la perspectiva transnacional.

\section{Bibliografía}

Anderson, Benedict (1983). Imagined Communities. Londres: Verso.

BAUBÖCK, R. (1994). Transnational Citizenship. Membership and Rights in International Migration. Aldershot: Elgar.

DoÑA, Cristián (2004). «Transnacionalismo y nuevas perspectivas de integración». La iniciativa de comunicación, marzo de 2004, p. 124-133. 
CAGLAR, Ayse (2001). "Constraining metaphors and the transnationalisation of spaces in Berlin». Journal of Ethnic and Migration Studies, núm. 27, 4, p. 601-613.

CONRADSON, D.; LATHAM, A. (2005). «Transnacional Urbanism: Attending to Everyday Practices and Mobilities». Journal of Ethnic and Migration Studies, vol. 31, 2, p. 227-233.

FAIST, Thomas (1999a). «Transnationalization in Internnational Migration: implications for the study of Citizenship and Culture». Working Paper WPTC-99-08 of Transnational Communities Programme. ISCA, University of Oxford. Consultado en: <www.transcom.ox.ac.uk>.

- (1999b). «Developing transnational social spaces: the Turkish German example». En: PRIES, L. (comp). Migration and Transnational Social Spaces. Aldershot: Ashgate, p. 36-72.

FitzGerald, David (2000). Negotiating Extre-Territorial Citizenship: Mexican Migration and the Transnational Politics of Community. Center for Comparative Immigration Studies. University of California.

- (2004). "Beyond "transnationalism": Mexican hometown politics at an American labour union». Ethnic and Racial Studies, vol. 27, núm. 2, p. 228-247.

Grillo, R. D. (2001). «Transnational Migration and Multiculturalism in Europe». Working Paper WPTC-01-08 of Transnational Communities Programme. ISCA, University of Oxford. Consultado en: <www.transcom.ox.ac.uk>.

GuARNizO, L. E. (2003). "The economics of transnational living». International Migration Review, vol. 37, núm. 3, p. 666-699.

Guarnizo, L. E.; Portes, A.; HALLER, W. (2003). «Assimilation and transnationalism: determinants of transnational political action among contemporary migrants». AJS, vol. 108, 6, p. 1211-1248.

KASTORYANO, Riva (2002). "The Reach of Transnationalism». En: HERSHBERG, E.; MoOre, K. Critical Views of September 11: Analyses from Around the World. W.W. Norton \& Company.

- (2003). "Transnational Participation and Citizenship: Immigrants in European Union». National Europe Centre Paper, núm.64.

KeARneY, Michael (1991). «Borders and boundaries of state and self at the end od empire». Journal of Historical Sociology, núm. 4, 1, p. 52-74.

- (1995). "The local and the global: the anthropology of globalization and Transnationalism». Annual Review of Antrhopology, núm. 24, p. 547-565.

KIVISTO, Peter (2001). «Theorizing transnational immigration: a critical review of current efforts». Ethnic and Racial Studies, vol. 24, núm. 4, p. 549-577.

LANDLOT, Patricia (2001). «Salvadoran economic transnationalism: embedded strategies for household maintenance, immigrant incorporation and entrepeneurial expansion». Global Networks, núm. 1, 3, p. 217-241.

- (2003). «El transnacionalismo político y el derecho al voto en el exterior. El caso de El Salvador y sus migrantes en Estados Unidos». En: CALDERÓN, L. (ed.). Votar en la distancia: la extensión de derechos políticos a migrantes: Experiencias comparadas. México, p. 301-323.

LUCASSEN, Leo (2004). «Is Transnationalism compatible with assimilation? Examples from Western Europe since 1850». Paper presented for the Conference Migranst, Nations and Citizenship. CRASSH. University of Cambridge.

MENDOZA, Cristóbal (2005). «El espacio fronterizo en la articulación de espacios sociales transnacionales: una reflexión teórica y unos apuntes empíricos». Seminario Internacional Problemas y desafios de la migración y el desarrollo en America, abril 
de 2005, Cuernavaca. Consultado en: <http://www.meme.phpwebhosting.com/ $\sim$ migracion/modules/seminarioe/mendozacristobal.pdf $>$.

MrTCHELL, Katharyne (1993). «Multiculturalism, or the United Colors of Capitalism?». Antipode, núm. 25(4), p. 263-294.

ONG, AIHWA (1997). Ungrounded Empires: The Cultural Politics of Modern Chinese Transnationalism (co-edited with Donald Nonini). Nueva York: Routledge

PORTES, Alejandro (1999). "Conclusion: towards a new world: the origins and effects of transnational activities». Ethnic and Racial Studies, vol. 22, núm. 2, p. 463-477.

- (2003). «Conclusion: Theoretical Convergencies and Empirical Evidence in the Study of Immigrant Transnationalism». International Migration Review, vol. 37, p. 874-892.

PorTeS, A.; GuarnizO, L. E.; LANDLOT, P. (1999). «The study of Transnationalism: pitfalls and promise of an emergent research field». Ethnic and Racial Studies, vol. 22, núm. 2, p. 217-237.

Portes, A.; Haller, W.; Guarnizo, L. E. (2002). «Transnational entrepeneurs: the emergente and determinants o fan alternative form of immigrant economic adaptation». American Sociological Review, núm. 67, p. 278-298.

Portes, A.; EsCOBAR, C.; WALTON, A. (2006). Immigrant Transnational Organizations and Development: A Comparative Study.

Smith, M. P.; Guarnizo, L. E. (1998). "The Locations of Transnationalism». En: GuarnizO, L. E.; Smith, M. P. (eds.), Transnationalism from Below. Comparative Urban and Community Research, vol. 6, p. 3-34.

SOYSAL, Y. N. (1994). Limitis of Citizenship. Migrants and Postnational Membership in Europe. The University of Chicago Press.

TAYLOR, J. E. (1999). «The new economics of labour migration and the role of remittances in the migration process». International Migration, núm. 37, 1, p. 63-88.

Vertovec, Steven (1999). "Conceiving and Researching Transnationalism». Ethnic and Racial Studies, vol. 22, núm. 2.

- (2001). "Transnational Challenges to the "new" Multiculturalism». Working Paper WPTC-01-06 of Transnational Communities Programme. ISCA, University of Oxford. Consultado en: <www.transcom.ox.ac.uk>.

- (2004). Trends and Impacts of Migrant Transnationalism. Centre on Migration, Policy and Society. University of Oxford. Working paper, núm. 3.

WALDINGER, R.; FITZGERALD, D. (2004). «Transnationalism in Question». American Journal of Sociology, núm. 109, 5, p. 1177-1195.

Werbner, Pnina (2000). «Divided Loyalties, Empowered Citizenship? Muslims in Britain». Citizenship Studies, vol. 4, núm. 3, p. 307-324. 